\title{
Nuevo cuestionario de emociones positivas para niños
}

\author{
Laura Beatriz Oros
}

CIIPME-UAP-CONICET; Instituto Superior Adventista de Misiones (Argentina)

\begin{abstract}
Resumen: El propósito de este trabajo fue diseñar y validar un nuevo instrumento para medir la experiencia emocional positiva de niños de 8 a 12 años de edad. La muestra piloto estuvo conformada por 445 niños $(n=$ 221) y niñas $(n=224)$, con una media de edad de 9.54 años $(D T=1.25)$, pertenecientes a diferentes provincias argentinas. La muestra definitiva estuvo integrada por 1046 niños $(n=540)$ y niñas $(n=505)$ con un promedio de edad de 9.74 años $(D T=1.33)$. Luego de ser analizado según diferentes parámetros de validez y fiabilidad, el cuestionario definitivo quedó conformado por 23 ítems y cuatro dimensiones: (a) alegría y gratitud $(a=.92)$; (b) serenidad $(a=.75)$; (c) simpatía $(a=.64)$; $\mathrm{y}$ (d) satisfacción personal $(a=$ .71). El Alpha de Cronbach para la escala total fue de .90. Los resultados del estudio psicométrico fueron adecuados, observándose un buen poder discriminativo de los ítems, una elevada consistencia interna y una estructura factorial coherente.

Palabras clave: Evaluación; cuestionario; emociones positivas; niños.
\end{abstract}

\section{Introducción}

Las emociones positivas, que en términos generales se suelen identificar como experiencias de bienestar, satisfacción o placer (Lucas, Diener y Larsen, 2003), están recibiendo cada vez mayor atención debido a su potencial efecto positivo sobre la salud. Estudios correlacionales y experimentales confirman que la experiencia frecuente de emociones como la alegría, la serenidad, la simpatía, la gratitud y la satisfacción favorecen el funcionamiento físico y psicosocial en diferentes etapas evolutivas (Boehm y Lyubomirsky, 2008; Fredrickson, 2000; Fredrickson y Joiner, 2002; Johnson, Waugh y Fredrickson, 2010; Martin, Puhlik-Doris, Larsen, Gray, y Weir, 2003; Ren, Hu, Zhang y Huang, 2010).

A diferencia de lo que ocurre en los países anglosajones, en Iberoamérica aún son relativamente escasas las investigaciones que se han realizado sobre este tópico. A pesar de ello, es posible detectar intentos rigurosos de operacionalización y estudio de las emociones positivas en países como Brasil, Perú, Chile, Venezuela, Ecuador, Bolivia y Argentina. Una rápida revisión bibliográfica indica que las emociones positivas y el bienestar psicológico/felicidad en América Latina han sido estudiados principalmente en adolescentes y adultos, con relación a: resiliencia y factores protectores (Cingolani y Méndez Quiñones, 2007; Lillo, 2006; Mustaca, Kamenetzky y Vera-Villarroel, 2010; Salgado Levano, 2009), procesos de salud-enfermedad (Porro Conforti y Andrés, 2011), variables socioculturales y económicas (Alves de Souza y Ferreira Dela Coleta, 2009; Lacerda Teixeira Pires y Alonso, 2008; Marín Romo, 2009; Reyes-García, et al. 2010; Vera Noriega, Laborín Álvarez, Córdova Moreno y Parra Armenta 2007); entornos académicos (Dela Coleta y Ferreira Dela Coleta, 2006, 2007; Mascarenhas y Peluso, 2011), inter-

* Dirección para correspondencia [Correspondence address]: Laura Beatriz Oros. Avenida Rusia 410. (3315) Leandro N. Alem, Misiones (Argentina). E-mail: lauraorosb@gmail.com
Title: A new questionnaire to assess positive emotions in children.

Abstract: The purpose of this study was to design and validate a new instrument to measure the positive emotional experience of children 8 to 12 years of age. The pilot sample consisted of 445 children $(n=221)$ and girls $(n=224)$, with a mean age of 9.54 years $(S D=1.25)$, belonging to different provinces of Argentina. The final sample consisted of 1046 children $(n=$ $540)$ and girls $(n=505)$ with an average age of 9.74 years $(S D=1.33)$. After being analyzed according to different parameters of validity and reliability, the final questionnaire was composed of 23 items and four dimensions: (a) joy and gratitude $(\alpha=.92)$, (b) serenity $(\alpha=.75)$, (c ) sympathy ( $\alpha=$ $.64)$, and (d) personal satisfaction $(\alpha=.71)$. The Cronbach alpha for the total scale was .90. Psychometric Study results were appropriate, with a good discriminative power of items, high internal consistency and consistent factor structure.

Key words: Assessment; questionnaire; positive emotions; children.

venciones psicoterapéuticas y psicoeducativas (Arias, Sabatini y Soliverez, 2011; Camacho, 2005; Chazenbalk, 2005; Moyano, 2011), variables familiares (Meier y Oros, 2012), personalidad (Regner, 2009a), y desempeño social (de la Vega y Oros, 2013; Regner, 2009b; Regner, 2011), entre otros.

Con respecto a la evaluación de las emociones positivas, los avances se han dirigido principalmente a la población adulta, encontrándose muy pocas escalas aplicables desde la niñez, realidad que también se ve reflejada fuera los límites de Latinoamérica. Algunos intentos latinoamericanos para evaluar afectos positivos y constructo relacionados en adultos incluyen la Escala de emociones positivas (Schmidt, 2008), la Escala de Felicidad de Lima (Alarcón, 2006) y su adaptación a adultos mayores venezolanos (Arraga Barrios y Sánchez Villarroel, 2010), la propuesta de Castro Solano (2011b) para evaluar la felicidad desde el enfoque de las tres rutas del bienestar psicológico, el Cuestionario de Estilos de Humor (Lillo, 2006), el Cuestionario de Emociones Positivas (Regner, 2009a), la Escala de Bienestar Subjetivo para adultos brasileños (Albuquerque y Tróccoli, 2004) y el Cuestionario de Bienestar Psicológico (BIEPS) basado en el modelo de Ryff (Castro Solano, Brenlla y Casullo, 2002; Casullo y Castro Solano, 2000), entre otras. Algunas de estas escalas son aplicables desde la adolescencia y todas presentan un buen funcionamiento psicométrico.

Para evaluar las emociones positivas en población infantil existen algunas escalas argentinas que han probado ser confiables y válidas. Entre ellas se cuentan la Positive Affect and Negative Affect Schedule versión niños (PANAS-C), propuesta originalmente por Laurent et al. (1999) y validada a la argentina por Schulz de Begle, Lemos y Richaud de Minzi (2009). Mediante 30 ítems esta escala proporciona un valor independiente para afectos positivos y negativos.

Para evaluar la gratitud se dispone del cuestionario de gratitud para niños (Cuello y Oros, 2011), que incorpora en su diseño la propuesta de McCullough, Emmons y Tsang (2002), que ha resultado válida y confiable al ser aplicada en 
población infantil (Froh y Emmons, 2007), y se basa también en las subescalas argentinas de gratitud desarrolladas por Schmidt (2008) y Regner (2009a) para adolescentes y adultos, respectivamente.

También existe una escala para evaluar la simpatía en niños (Oros, 2006) basada en los conceptos teóricos de Lazarus (2000) y Eisenberg (1991) y en la propuesta operacional de Bryant (1982, adaptada al español por Mestre Escrivá, Pérez-Delgado, Frías Navarro y Samper García, 1999) para medir específicamente la dimensión de sintonía emocional.

La escala ESI (Oros, 2001) evalúa la serenidad infantil desde la perspectiva de un informante externo (padres, cuidadores, docentes). Se trata de una escala muy breve que puede ser utilizada con fines complementarios a otras medidas, y que arroja información acerca de cuán sencillo o complejo resulta a los niños regular sus emociones y relajarse en momentos de estrés.

También existe una escala pictórica de alegría para niños pequeños (Oros, 2008b), la cual presenta una secuencia de siete expresiones faciales graduadas desde la más triste a la más alegre con una de las cuales debe identificarse el/la niño/niña. Greco (2010) realizó una contribución interesante a esta escala al adicionar preguntas para indagar atribuciones y comportamientos ligados a la experiencia emocional de alegría.

Todas estas escalas reportan buenas propiedades psicométricas y un excelente ajuste con los modelos teóricos existentes pero su utilidad se restringe a la evaluación de una única emoción positiva o, como en el caso de la PANAS-C, a la obtención de un valor general e indiscriminado de afectividad positiva. Además, debido a que estas escalas presentan estilos y formatos muy dispares se dificulta la posibilidad de establecer comparaciones válidas entre ellas o de generar mediante su uso conjunto un índice general de emocionalidad positiva. Hasta el momento no se conoce la existencia de un autoinforme para niños argentinos que permita obtener un perfil emocional positivo considerando varias emociones simultáneamente. El presente trabajo se propuso llenar este vacío mediante la construcción y validación de un cuestionario autoadministrable basado en las medidas preexistentes para evaluar alegría, gratitud, serenidad, simpatía y satisfacción personal en niños argentinos de 8 a 12 años de edad. Tal como fue descripto, las escalas precedentes fueron desarrolladas tomando en consideración modelos teóricos y empíricos de probada utilidad, razón que justificó su empleo como punto de referencia para la construcción de este nuevo instrumento.

Existen otras numerosas emociones positivas que podrían evaluarse en la niñez; tal es el caso del humor, el interés, la esperanza, el entusiasmo, el amor, el vigor, etc. Sería poco realista pretender cubrir con un solo cuestionario todas las emociones positivas que tienen relevancia práctica en el periodo evolutivo que se estudia, aún en el caso de que esta información estuviera disponible y consensuadamente aceptada. En esta oportunidad hemos seleccionado apenas una muestra del dominio general de emociones positivas toman- do como criterio de inclusión que hayan demostrado ser empíricamente relevantes en estudios previos con niños argentinos, y que se disponga de antecedentes métricos válidos y confiables para medirlas (Hendrie y Lemos, 2011; Oros 2008b, 2009a; 2009b; 2011; Oros y Richaud, 2011; Schulz de Begle, 2009). A continuación se presentarán las definiciones conceptuales de las cinco emociones seleccionadas.

La alegría es una intensa emoción positiva desencadenada por situaciones de vida satisfactorias y por progresos significativos en dirección a las metas personales. Se refiere a un estado general de contentamiento, diversión y regocijo, cuya máxima expresión es la risa (Lazarus, 2000).

La gratitud es una emoción empática de gran relevancia social (Lazarus, 2000). Se experimenta gratitud cuando: (a) se obtiene un beneficio que es evaluado como positivo, (b) ese beneficio no se ha conseguido por el esfuerzo propio, sino que (c) ha sido otorgado intencionalmente por un benefactor (Emmons, McCullough y Tsang, 2003). El sentimiento genuino de gratitud retroalimenta las conductas prosociales, puesto que una persona agradecida sentirá deseos de retribuir a su benefactor, quien ha mostrado altruismo y prosocialidad antes.

La serenidad es una emoción profunda que involucra paz y confianza. Puede experimentarse independientemente de los eventos externos, incluso en situaciones de adversidad (Connors, Toscova y Tonigan, 1999; Roberts y Cunningham, 1990). La serenidad facilita el afrontamiento al estrés (Connors, Toscova y Tonigan, 1999) y permite tomar distancia de aquellos pensamientos que generan inquietud y/o angustia. La regulación emocional y la capacidad de relajación constituyen elementos clave de la habilidad para serenarse.

La simpatía constituye el componente afectivo de la empatía. Envuelve la capacidad de sintonizar con la emoción de los demás y la inclinación a ayudar. La simpatía es una reacción emocional vicaria basada en la preocupación por la condición emocional del otro, que involucra sentimientos de pena e interés y comprende el deseo de que el otro se sienta mejor (Eisenberg, 1991; Kienbaum, Volland y Ulich, 2001). De acuerdo a Lazarus (2000), éste es un concepto intercambiable con el de compasión.

La satisfacción personal, también llamada orgullo, es la emoción positiva que acompaña los procesos de valoración, promoción del self y autoatribución de méritos valiosos (Lazarus, 2000). Es una respuesta afectiva relacionada a la conformidad y a la aceptación de uno mismo. En este sentido, el concepto de satisfacción personal está estrechamente ligado al de autoestima. Entendida así, la satisfacción personal se diferencia del concepto de satisfacción con la vida, el cual es un componente principalmente cognitivo del bienestar subjetivo, y se refiere al juicio global acerca de la vida (Diener y Larsen, 1993). 


\section{Método}

\section{Participantes}

Se trabajó con una muestra piloto de 445 niños $(n=221)$ y niñas $(n=224)$ escolarizados, con una media de edad de 9.54 años $(D T=1.25)$, de nivel socioeconómico medio, pertenecientes a las provincias argentinas de Entre Ríos, Buenos Aires y Tucumán. La muestra definitiva estuvo integrada por 1046 niños $(n=540)$ y niñas $(n=505)$ con un promedio de edad de 9.74 años $(D T=1.33)$, que residían en las mismas provincias y asistían a escuelas primarias, tanto públicas como privadas. Ambas muestras fueron seleccionadas de manera no probabilística, intencional, bajo la condición de que los niños presentaran el consentimiento informado de sus cuidadores primarios.

\section{Instrumentos}

Cuestionario Infantil de Emociones Positivas (CIEP)

Tomando como base la bibliografía especializada en la temática, se conformó un cuestionario con 40 enunciados para operacionalizar la alegría, la serenidad, la simpatía, la satisfacción personal y la gratitud. Para la redacción de los enunciados se siguieron las recomendaciones de Matesanz Nogales (1997) en cuanto a sencillez, claridad y precisión de los ítems. Las frases se redactaron de modo aseverativo, en primera persona, presentan una longitud adecuada (menos de 12 términos por ítem) y describen un solo comportamiento o experiencia a la vez. Algunos de los ítems para evaluar simpatía y serenidad fueron extraídos textualmente de la Escala de Simpatía para niños (Oros, 2006) y de la Escala de Gratitud Infantil (Cuello y Oros, 2011), respectivamente. Cada enunciado se puede responder con una de tres posibles opciones: (3) si, (2) más o menos, o (1) no, según el grado de acuerdo con la frase que se expresa. Luego de someter esta versión preliminar a estrictos controles de confiabilidad y validez, se conservó poco más del 50\% de los ítems, por ser los que mostraron el mejor funcionamiento psicométrico.

\section{Escala de Deseabilidad Social Infantil (EDESI)}

La EDESI (Lemos, 2005) fue utilizada como un parámetro para evaluar la validez interna, con el fin de descartar la posibilidad de que las puntuaciones obtenidas con el CIEP se vieran más explicadas por la tendencia infantil a atribuirse cualidades socialmente deseables que por la verdadera experiencia emocional de los niños.

\section{Procedimiento}

Se solicitó la autorización a los directivos y docentes de las diferentes escuelas para poder tomar contacto con los alumnos en el horario habitual de clases. La aplicación de los cuestionarios se llevó a cabo durante el año 2011 por perso- nal debidamente entrenado. La participación de los niños fue voluntaria y se realizó previo consentimiento informado de sus padres o tutores legales. El tiempo de administración fue de entre 15 a 20 minutos.

\section{Análisis de datos}

Se analizaron las siguientes propiedades psicométricas del CIEP: (a) el poder discriminativo de los ítems, mediante la prueba $t$ de Student para grupos contrastantes (Anastasi y Urbina, 1990; Namakforoosh, 2000); (b) la consistencia interna, mediante el coeficiente Alpha de Cronbach, el índice de correlación Spearman-Brown para división por mitades y las correlaciones corregidas ítem-subescalas; (c) la estabilidad temporal, mediante la correlación bivariada de Pearson entre aplicaciones sucesivas; (d) la validez de la escala, a través de un Análisis Factorial Exploratorio ( $A F E$ ) con el método de ejes principales y rotación varimax; y a partir de las correlaciones entre el CIEP y la EDESI. Todos los análisis fueron realizados mediante el programa estadístico SPSS 18.0.

Si bien es cierto que al trabajar con escalas ordinales es recomendable utilizar modelos de análisis no lineales, basados en correlaciones policóricas (Ferrando y AnguianoCarrasco, 2010; Richaud de Minzi, 2005), se ha decidido realizar un $A F E$ atendiendo a que, salvo en casos extremos (por ejemplo cuando los datos son marcadamente asimétricos), este método (y otros similares, como el $A C P$ ) tiende a producir estimaciones razonables, siendo utilizado habitualmente por la comunidad científica como una aproximación exploratoria para el estudio de escalas tipo Likert que evalúan variables afectivas y de personalidad infantil (e.g. Carrasco Ortíz, Holgado Tello y del Barrio Gándara, 2005; Mesurado, 2008; Sandín, Chorot, Santed y Valiente, 2002; Sandín, Valiente y Chorot, 2009; Rodríguez Villagra, Padilla Mora, y Fornaguera Trías, 2010, Ugarriza Chávez y Pajarés del Águila, 2005, etc.). Para analizar los supuestos de normalidad de los datos de este estudio se calcularon los índices de asimetría y curtosis encontrando valores que oscilan entre +1 y -1 para todas las variables, dando cuenta de una distribución que se acerca a la normal. El análisis de datos perdidos indicó que ninguno de los ítems presentaba porcentajes de omisión mayores al 5\%. Para la sustitución de los valores faltantes se utilizó el procedimiento de imputación por la media.

Debido a que no se dispone de fuertes hipótesis previas respecto al grado de asociación o independencia entre las emociones evaluadas, y estimando que algunas podrían llegar a comportarse independientemente de las otras, para la realización del AFE se decidió probar tanto la rotación oblicua como la ortogonal. Se encontró que si bien ambas rotaciones convergían en una solución común de igual cantidad de factores y mismo contenido teórico, la rotación varimax resultaba bastante más parsimoniosa, aproximando la solución factorial a una estructura simple y facilitando la interpretación de los factores. De acuerdo a los resultados de la rotación oblicua, las correlaciones entre los factores resultaron 
entre bajas y moderadas, razón que justificó también la aplicación de una rotación ortogonal (ver Resultados).

\section{Resultados}

\section{Prueba piloto de la versión preliminar}

El CIEP tuvo un buen funcionamiento psicométrico en la muestra piloto $(n=445)$. El poder discriminativo de los ítems, calculado a partir de la prueba t de diferencia de medias, fue adecuado y significativo para todos los elementos $(p$ $<.00)$, y la consistencia interna de la prueba completa resultó satisfactoria $(a=.84)$.

Para estudiar la estructura factorial se realizó un Análisis Factorial Exploratorio ( $A F E$ ) habiendo previamente empleado el test de esfericidad de Bartlett y la medida de adecuación muestral de Kaiser-Meier-Olkin $(K M O=.82$; Chi cuadrado de Bartlett= 1614.63; $p<.00$ ).

Cuando se utilizó el criterio de extracción del autovalor 1 se obtuvo una matriz sobrefactorizada, observándose 11 dimensiones, varias de las cuales resultaron residuales. $\mathrm{Al} \mathrm{em-}$ plear el criterio de la pendiente de Cattell emergieron 8 factores que igualmente resultaron ser excesivos ya que algunos incluían apenas una saturación significativa o sólo contenían ítems complejos. Por este motivo, se fue ajustando paulatinamente el número de factores, eliminando los triviales, hasta llegar a una estructura factorial clara, de cinco dimensiones explicables, congruentes con las cinco emociones que se pretendían evaluar.

Se descartaron de este análisis los ítems con saturaciones inferiores a .30, los ítems con marcada complejidad factorial (diferencia de saturación entre ítems menor a .10), y aquellos que se saturaron en un factor diferente al que habían sido propuestos, a excepción del ítem Casi siempre estoy relajado, conservando un total de 21 enunciados. Se decidió conservar este ítem para evaluar nuevamente su funcionamiento factorial en la prueba definitiva dado que aunque ha resultado complejo en este análisis preliminar, contribuye a aumentar la consistencia interna de la dimensión para la cual fue creado originalmente.

En el primer factor se incluyeron 5 ítems que evalúan alegría $(a=.70)$, en el segundo factor se agruparon 6 ítems que evalúan serenidad ( $a=.74)$, en el tercer factor se agruparon 5 ítems que evalúan simpatía $(a=.70)$, en el cuarto factor se incluyeron 3 ítems que evalúan satisfacción personal $(a=.68)$ y en el quinto factor se agruparon 2 ítems que evalúan gratitud $(a=.63)$. (Ver Tabla 1$)$.

Tabla 1. Estructura factorial del cuestionario en la muestra piloto.

\begin{tabular}{|c|c|c|c|c|c|}
\hline \multirow[t]{2}{*}{ Ítems } & \multicolumn{5}{|c|}{ Dimensiones } \\
\hline & F1 & F2 & F3 & F4 & F5 \\
\hline Soy una persona alegre & .58 & .04 & .12 & .20 & .14 \\
\hline Soy muy feliz & .62 & .08 & .23 & .11 & .09 \\
\hline Me divierto mucho con las cosas que hago & .33 & .20 & .04 & .16 & .16 \\
\hline Casi siempre estoy contento/a & .63 & .16 & .10 & .14 & .05 \\
\hline Casi siempre la paso bien & .53 & .22 & .07 & .00 & .05 \\
\hline Soy bastante tranquilo & .24 & .37 & .04 & .14 & -.06 \\
\hline Soluciono mis problemas con mucha tranquilidad & .25 & .36 & .13 & .21 & .23 \\
\hline Casi siempre estoy relajado & .42 & .35 & .18 & .00 & .03 \\
\hline La mayor parte de los días me siento en paz & .29 & .40 & .07 & .13 & .16 \\
\hline Aunque tenga problemas, igual mantengo la calma & .11 & .63 & .01 & .21 & .21 \\
\hline Me quedo tranquilo aunque no pueda hacer lo que me gusta & .11 & .59 & .08 & .19 & .09 \\
\hline Me pone triste ver a otra/o nena/e que no tiene con quien jugar & .17 & -.00 & .01 & .47 & .16 \\
\hline Si veo llorar a un nene/a me dan ganas de llorar a mí también & .06 & .19 & .09 & .51 & -.01 \\
\hline Me pongo muy mal si veo que alguien se lastima & .11 & .10 & .06 & .60 & .10 \\
\hline Cuando alguien está solo y aburrido me dan ganas de... & .07 & .22 & -.07 & .52 & .19 \\
\hline Si alguien está llorando me dan ganas de abrazarlo o consolarlo & .09 & .34 & .15 & .60 & .17 \\
\hline Me quiero mucho a mi mismo & .16 & .06 & .46 & .13 & .00 \\
\hline Siento que soy muy valioso & .13 & .15 & .74 & .06 & -.02 \\
\hline Siento que soy importante & .14 & -.00 & .78 & -.04 & .07 \\
\hline Me gusta devolver favores & .18 & .18 & .01 & .17 & .55 \\
\hline Siempre que puedo, devuelvo los favores que recibo & .11 & .11 & .03 & .24 & .75 \\
\hline
\end{tabular}

F1: Alegría; F2: Serenidad; F3: Satisfacción; F4: Simpatía; F5: Gratitud.

\section{Funcionamiento psicométrico del CIEP en la mues- tra definitiva}

El funcionamiento factorial de la escala fue nuevamente puesto a prueba con una muestra de 1046 niños y niñas. Debido a que el factor Gratitud sólo incluyó dos ítems en la prueba piloto, se optó por incluir tres ítems más de gratitud para evaluar su comportamiento en la muestra definitiva. Estos ítems fueron: Me gusta agradecerle a la gente; V aloro cuando los demás me ayudan y Estoy agradecido con varias personas, por lo que bacen por mí. La nueva estructura factorial (KMO = .94; Chi cuadrado de Bartlett $=9491,401 ; p<.00)$ se vio levemente modificada respecto al análisis inicial, ya que se aglutinaron la alegría y la gratitud en una sola dimensión, se eliminó el 
ítem Me pone triste ver a otra/ o nenal e que no tiene con quien jugar, porque no alcanzó la saturación mínima estipulada, y se presentaron dos ítems complejos (Me quiero mucho a mi mismo y Cuando alguien está solo y aburrido me dan ganas de acercarme y jugar con él) correspondientes a las dimensiones de satisfacción personal y simpatía, respectivamente. Para la clasificación de estos últimos se decidió adoptar un criterio teórico tomando en consideración las dimensiones para las cuales habían sido formulados y en las que habían saturado con valores elevados en la prueba de pilotaje. Además, se comprobó que estos ítems no contribuían a la consistencia interna de las dimensiones alternativas, o incluso disminuían sus valores de Alpha de Cronbach. Tomando en consideración que en este nuevo análisis factorial el promedio de las saturaciones principales era elevado (por encima de .60); se decidió conservar para cada factor sólo a aquellos ítems con saturaciones iguales o superiores a .40 .
La extracción de estos cuatro factores estuvo apoyada por el gráfico de sedimentación que mostró claramente la existencia de 4 dimensiones: (a) alegría y gratitud, con 10 ítems $(a=.92)$; (b) serenidad, con 6 ítems $(a=.75)$; (c) simpatía, con 4 ítems $(a=.64)$; y (d) satisfacción personal, con 3 ítems $(a=.71)$ (Ver Tabla 2). Si se refactoriza el primer factor, la alegría y la gratitud conforman dimensiones independientes conservando aún valores elevados de consistencia interna ( $a=.86$ y $a=.88$, respectivamente).

Los cuatro factores explican el $47.88 \%$ de la variancia total. El primer factor explica un $24.44 \%$ de la variancia total, el segundo un $10.60 \%$, el tercero un $7.06 \%$ y el cuarto un $5.79 \%$. Aunque los dos últimos factores inducen un porcentaje de variancia menor al 10\%, su inclusión queda justificada debido a la importancia teórica que revisten dentro del modelo y sus aceptables valores de consistencia interna.

Tabla 2. Estructura factorial del cuestionario y correlaciones ítem-subescalas en la muestra definitiva.

\begin{tabular}{|c|c|c|c|c|c|}
\hline \multirow[t]{2}{*}{ Ítems } & \multicolumn{4}{|c|}{ Dimensiones } & \multirow[b]{2}{*}{ Correl. ítem- dimensión } \\
\hline & F1 & F2 & F3 & F4 & \\
\hline Soy una persona alegre & .72 & .23 & .24 & -.01 & .74 \\
\hline Soy muy feliz & .67 & .28 & .29 & -.04 & .73 \\
\hline Me divierto mucho con las cosas que hago & .62 & .21 & .19 & .01 & .64 \\
\hline Casi siempre estoy contento/a & .50 & .36 & .26 & -.02 & .59 \\
\hline Casi siempre la paso bien & .61 & .36 & .25 & -.12 & .67 \\
\hline Soy bastante tranquilo & .08 & .54 & .03 & .13 & .47 \\
\hline Soluciono mis problemas con mucha tranquilidad & .16 & .50 & .05 & .19 & .48 \\
\hline Casi siempre estoy relajado & .07 & .59 & .17 & .02 & .48 \\
\hline La mayor parte de los días me siento en paz & .25 & .54 & .18 & .08 & .50 \\
\hline Aunque tenga problemas, igual mantengo la calma & .20 & .54 & -.01 & .23 & .52 \\
\hline Me quedo tranquilo aunque no pueda hacer lo que... & .13 & .46 & .07 & .23 & .45 \\
\hline Si veo llorar a un nene/a me dan ganas de llorar... & -.17 & .14 & .05 & .47 & .30 \\
\hline Me pongo muy mal si veo que alguien se lastima & .18 & .24 & .07 & .49 & .46 \\
\hline Cuando alguien está solo y aburrido me dan... & .49 & .20 & .04 & .45 & .43 \\
\hline Si alguien está llorando me dan ganas de abrazarlo... & .35 & .23 & .10 & .56 & .54 \\
\hline Me quiero mucho a mi mismo & .45 & .10 & .40 & .06 & .41 \\
\hline Siento que soy muy valioso & .20 & .13 & .74 & .12 & .60 \\
\hline Siento que soy importante & .22 & .14 & .70 & .07 & .58 \\
\hline Me gusta devolver favores & .62 & .24 & .05 & .23 & .63 \\
\hline Siempre que puedo, devuelvo los favores que recibo & .69 & .16 & .09 & .23 & .67 \\
\hline Me gusta agradecerle a la gente & .77 & .17 & .09 & .16 & .75 \\
\hline Valoro cuando los demás me ayudan & .82 & .04 & .09 & .11 & .75 \\
\hline Estoy agradecido con varias personas, por lo... & .81 & .04 & .13 & .11 & .75 \\
\hline
\end{tabular}

F1: Alegría y Gratitud; F2: Serenidad; F3: Satisfacción; F4: Simpatía.

Las intercorrelaciones entre dimensiones resultaron bajas o moderadas (oscilando entre $r=-.01$ y $r=.37$ para $n=$ 1046), lo cual apoya la relativa independencia entre las emociones. La dimensión de alegría-gratitud obtuvo las correlaciones más altas, asociándose moderadamente con la serenidad $(r=.37)$ y la satisfacción personal $(r=.36)$.

El coeficiente Alpha de Cronbach para la prueba completa fue de $.90 \mathrm{y}$ el coeficiente de Spearman-Brown para mitades de longitud desigual fue de .82. Para evaluar la estabilidad temporal, 205 niños respondieron el cuestionario en dos oportunidades, con una diferencia de entre dos y tres meses entre ellas. La correlación test-re-test fue moderadamente al- ta para el cuestionario completo, alcanzando un valor de $r=$ $.71(p<.00)$. Las correlaciones test-re-test para las subescalas resultaron algo más bajas oscilando entre $r=.54(p<.00)$ para satisfacción personal, dimensión que incluye sólo tres ítems, y $r=.66(p<.00)$ para simpatía.

En la Tabla 2 se pueden apreciar las correlaciones corregidas ítem-subescala (exceptuado el propio ítem). Todas las correlaciones han resultado entre moderadas y moderadamente altas confirmando que en general los ítems tienden a reflejar los dominios a los que pertenecen. Las correlaciones más elevadas corresponden a los ítems que evalúan alegría y gratitud (promedio de correlaciones $=.69$ ) mientras que las 
más débiles corresponden a la simpatía (promedio de correlaciones $=.43$ ).

La prueba completa presentó una correlación baja $(r=$ $.26, p<.05)$ con la deseabilidad social. La misma tendencia se observó respecto a las dimensiones alegría-gratitud $(r=$ $.18, p<.11)$; serenidad $(r=.25, p<.02)$; simpatía $(r=-.02, p$ $<.84)$ y satisfacción personal $(r=.24, p<.03)$. Esto indicaría que el CIEP permite recabar información válida acerca de la experiencia emocional positiva de los niños independientemente de la intención psicológica que puedan presentar algunos de ellos a atribuirse cualidades socialmente deseables aunque no las presenten, como ser simpáticos con sus compañeros, alegres o tranquilos.

\section{Aspectos de la puntuación}

Para obtener la puntuación del instrumento se asigna valor 3 a la respuesta afirmativa, valor 2 a la respuesta intermedia y valor 1 a la respuesta negativa. Si se desea calcular un valor total de emocionalidad positiva se suman los 23 ítems de la escala. Con el fin de conseguir valores independientes para cada tipo de emoción se suman los ítems 1, 2, 4, 10,12, $13,15,18,22$ y 23 para la dimensión alegría-gratitud; los ítems 3, 5, 9, 14, 17 y 19 para la dimensión serenidad; los ítems 6, 7, 11 y 21 para la dimensión simpatía; y los ítems 8, 16 y 20 para la dimensión satisfacción personal. (Ver Anexo).

\section{Discusión y conclusiones}

Si bien ha proliferado en los últimos años el estudio de las emociones positivas, el foco de interés se ha centrado prioritariamente en la población adulta y adolescente, quedando la franja infantil un tanto descuidada. Este estudio se propuso desarrollar un nuevo instrumento para evaluar emociones positivas en niños, el cual pudiera proporcionar no sólo un valor general de emocionalidad positiva sino también un perfil de diferentes emociones particulares.

De acuerdo con los resultados obtenidos, todos los ítems que componen la escala tienen la capacidad de discriminar significativamente entre los niños que presentan valores elevados de emocionalidad positiva y aquellos que muestran puntajes bajos. Con relación a la fiabilidad de la prueba puede decirse que los coeficientes de consistencia interna resultan adecuados tanto para la prueba en su conjunto como para cada dimensión por separado. Dichos resultados son alentadores teniendo en cuenta que algunas dimensiones incluyen un número muy reducido de ítems. Si bien la correlación test-re-test no es muy elevada, debe considerarse que las

\section{Referencias}

Alarcón, R. (2006). Desarrollo de una escala factorial para medir la felicidad. Revista Interamericana de Psicología, 40(1), 95-102.

Albuquerque, A. S., \& Tróccoli, B. T. (2004). Desenvolvimento de uma escala de bem-estar subjetivo. Psicologia: Teoria e Pesquisa, 20(2), 153-164. respuestas de los niños suelen ser más inestables que las de los adultos (Lemos, 2006) y que el período que se sucedió entre una evaluación y otra fue quizá demasiado extenso para los niños de edades más bajas (dos meses y medio de diferencia en promedio).

El análisis factorial mostró la existencia de cuatro dimensiones: (a) alegría y gratitud, (b) serenidad, (c) simpatía y (d) satisfacción personal. Aunque hubiera sido esperable que las emociones alegría y gratitud conformaran factores independientes, el hecho de que se hayan fusionado demuestra que el sentimiento de gratitud está, por definición, impregnado de la experiencia de alegría. Es natural que el niño que valora o aprecia un bien recibido y que siente deseos de retribuirlo a su benefactor experimente un sentimiento genuino de alegría y bienestar.

Por otra parte, también se considera valiosa la información que se aporta con respecto a la deseabilidad social ya que no siempre se estima su inclusión en estudios de validación psicométrica, siendo que esta tendencia psicológica puede conducir a resultados distorsionados que, en extremo, pueden incluso invalidar una medición psicológica (Lemos, 2005).

Con el fin de ampliar las evidencias de validez, actualmente se está administrando la versión definitiva del cuestionario a una muestra diferente de niños con el doble propósito de: (a) analizar la estabilidad de la estructura factorial a través de un Análisis Factorial Confirmatorio, y (b) estudiar hipótesis sobre su validez de constructo mediante la aplicación simultánea de otros instrumentos que evalúan diferentes procesos emocionales, cognitivos y sociales en la niñez media. Además, y a partir de una muestra representativa de niños argentinos, se generarán los valores normativos del CIEP con el fin de ofrecer un criterio de comparación para futuros estudios.

Debido a que la evaluación de otras emociones positivas en niños sigue representando un gran desafío para la ciencia psicológica, se sugiere trabajar en la construcción o adaptación de escalas que ofrezcan una aproximación teórica y práctica a otras emociones positivas menos estudiadas en niños como son el amor, el humor y la esperanza.

Agradecimientos.- Este trabajo fue realizado mediante un subsidio de la Facultad de Humanidades, Educación y Ciencias Sociales de la Universidad Adventista del Plata. Se agradece especialmente la contribución de la Lic. Mónica Serppe y de los asistentes de investigación quienes realizaron tareas de administración de pruebas y tabulación de datos: Melissa Valega, Maricel Leal, Viviana Martínez, Amalia Apablaza, Gisela Yanzón, Florencia Locatelli, Yanina Jourdán, Valeria Twerda y Magalí Baier.

Alves de Souza, A. \& Ferreira Dela Coleta, M. (2009). Significado e correlatos do sentimento de felicidade. Horizonte Científico, 3(2), 1-30. Anastasi, A. y Urbina, S. (1990). Test psicológicos. México: Prentice Hall. 
Arias, C. J., Sabatini, M. B., \& Soliverez, V. C. (2011, Noviembre). El trabajo con aspectos positivos para la promoción de la salud en la vejez. Memorias del Tercer Congreso Internacional de Investigación y Práctica en Psicología (pp. 14-16), Buenos Aires: UBA.

Arraga Barrios, M. V., \& Sánchez Villarroel, M. (2010). Bienestar Subjetivo en Adultos Mayores Venezolanos. Revista Interamericana de Psicología, 44 (1), 12-18.

Boehm, J. K. \& Lyubomirsky, S. (2008). Does Happiness Promote Career Success? Journal of Career Assessment, 16(1), 101-116.

Bryant, B. K. (1982). An Index of Empathy for Children and Adolescents. Child Development, 53, 413-425.

Camacho, J. M. (2005). El humor y la dimensión creativa en la psicoterapia. Psicodebate. Psicología Cultura y Sociedad, 6, 45-58.

Castro Solano, A. (2011a, Noviembre). La Psicología Positiva en Latinoamérica y en el mundo. Comunicación presentada en el Sexto Encuentro Iberoamericano de Psicología Positiva, Buenos Aires, Argentina.

Castro Solano, A. (2011b). Las rutas de acceso al bienestar. Relaciones entre bienestar hedónico y eudaemónico. Revista de Evaluación y Diagnóstico Psicológico, 31(1), 37-58

Castro Solano, A., Brenlla, M., and Casullo, M. M. (2002). Evaluación del bienestar psicológico en adultos argentinos. En M. M. Casullo (Ed.), Evaluación del bienestar psicológico en Iberoamérica (pp. 93-100). Buenos Aires: Paidós.

Casullo, M. M. \& Castro Solano, A. (2000). Evaluación del bienestar psicológico en estudiantes adolescentes argentinos. Revista de Psicología de la Pontificia Universidad Católica de Perú, 18(2), 36-68.

Chazenbalk, L. (2005). El valor del humor en el proceso psicoterapéutico. Psicodebate. Psicologia, Cultura y Sociedad, 6, 73-84.

Cingolani, J. M. \& Méndez Quiñonez, A. (2007). El bienestar psicológico en la ciudad de Mar del Plata. Perspectivas en Psicología. Revista de Psicología y Ciencias Afines, 4(1), 59-65.

Connors, G. J., Toscova, R. T. \& Tonigan, S. J. (1999). Serenity. En William R. Miller (Ed.) Integrating Spirituality into Treatment. Resources for Practitioners, 235-250. Washington DC: American Psychological Association.

Cuello, M. \& Oros, L. B. (2011, inédito). Cuestionario de gratitud para niños. Centro Interdisciplinario de Psicología Matemática y Experimental. Buenos Aires: Argentina.

De la Vega, N. \& Oros, L. B. (2013). El rol de las emociones positivas empáticas en el comportamiento social de los adolescentes. Psicodebate, 13(1), 4-18.

Dela Coleta, J. A. y Ferreira Dela Coleta, M. (2006). Felicidade, bem-estar subjetivo e comportamento acadêmico de estudantes universitarios. Psicologia em Estudo, 11(3), 533-539.

Dela Coleta, J. A. y Ferreira Dela Coleta, M. (2007). Felicidade e bem-estar subjetivo entre profesores universitarios. Revista de Estudos de Educasao, 9(2), 131-144.

Diener, E. \& Larsen, R.J. (1993). The experience of emotional Well-being. En M. Lewis y J.M. Haviland (Eds.), Handbook of emotions (pp. 404-415). Nueva York: Guilford Press.

Eisenberg, N. (1991). Values, sympathy and individual differences: toward an pluralism of factor influencing altruism and empathy. Psychological Inquiry, 2(2), 128-131.

Emmons, R. A., McCullough, M. E. \& Tsang, J. (2003). The Assesssment of Gratitude. En S. Lopez and C.R. Snyder. A Handbook of Models and Measures. Positive Psychological Assessment, 327-341. Washington, DC: American Psychological Association.

Fredrickson, B. L. (2000). Cultivating positive emotions to optimize health and well-being. Prevention \& Treatment, 3. Recuperado de http://www.unc.edu/peplab/publications/cultivating.pdf

Fredrickson B \& Joiner T. (2002). Positive Emotion Trigger Upward Spirals Toward Emotional Well-Being. Psychological Science, 13 (2), 172-175.

Giqueaux, G. \& Oros, L. (2008, octubre). Las emociones positivas como facilitadoras de la sociabilidad infantil. Sesión de cartel presentada en el XII Congreso Nacional de Psicodiagnóstico, La Plata, Argentina.

Greco, C. (2010). Emociones positivas: herramientas psicológicas para la promoción de la salud mental infantil en contextos de vulnerabilidad social. Manuscrito de Tesis Doctoral en Psicología. Universidad de San Luis, Argentina.

Hendrie, K. \& Lemos, V. (2011, noviembre). Simpatía y su incidencia en la conducta prosocial en niños de 6 y 7 años. Comunicación presentada en el $3^{\circ}$
Congreso Internacional sobre Investigación y Práctica Profesional en Psicología. Buenos, Aires, Argentina.

Johnson, K. J., Waugh, C. E., \& Fredrickson, B. L. (2010). Smile to see the forest: Facially expressed positive emotions broaden cognition. Cognition and Emotion, 24(2), 299-321

Kienbaum, J., Volland, C. \& Ulich, D. (2001). Sympathy in the context of mother-child and teacher-child relationships. International Journal of Behavioral Development 25(4), 302-309.

Lacerda Teixeira Pires, A. M. \& Molero Alonso, F. (2008). El prejuicio racial en Brasil: nuevas medidas y perspectivas. Psicologia \& Sociedade, 20(2), 267-276.

Laurent, J., Catanzaro, S., Joiner, T. E., Rudolph, K. D., Potter, K. I., Lambert, S., Osbome, L., y Gathright, T. (1999). A mea-sure of positive and negativo affect for chil-dren: Scale development and preliminaryvalidation. Psychological Assessment, 11,326-338.

Lazarus, R. S. (2000). Estrés y emoción. Manejo e implicaciones en nuestra salud. Barcelona: Desclée de Brouwer.

Lemos, V. (2005). Construcción y validación de una escala para la evaluación de la deseabilidad social infantil. Revista Interdisciplinaria, 22(1), 77-96.

Lemos, V. (2006). La deseabilidad social en la evaluación de la personalidad infantil. Suma Psicológica, 13(1), 7-17.

Lillo, P. R. (2006). Influencia de los estilos de humor sobre las estrategias de afrontamiento en Entre Ríos. Psicodebate. Psicología, Cultura y Sociedad, 7 , 95-118.

Lucas, R. E., Diener, E. \& Larsen, R. J. (2003). Measuring Positive Emotions. En S. J. Lopez \& C. R. Zinder (Eds.), Positive Psychological Assessment. A Handbook of Models and Measures (pp.201-218). Washington: American Psychological Association.

Marín Romo, R. F. (2009). Indicadores Subjetivos de Bienestar versus Indicadores Objetivos de Bienestar. Una aplicación para el caso de las nacionalidades y pueblos del Ecuador (Tesis de maestría no publicada). Facultad Latinoamericana de Ciencias Sociales. Ecuador.

Martin, R. A., Puhlik-Doris, P., Larsen, G., Gray, J. \& Weir, K. (2003). Individual differences in uses of humor and their relation to psychological well-being: Development of the Humor Styles Questionnaire. Journal of Research in Personality, 37 (1), 48-75.

Mascarenhas, S. A. \& Peluso, M. L. (2011). Indicadores do bem estar subjetivo de discentes do ensino superior no amazonas. Amazónica, 7(2), 77 87.

Matesanz Nogales, A. (1997). Evaluación estructurada de la personalidad. Madrid: Pirámide.

McCullough, M. E., Emmons, R. A., \& Tsang, J. A. (2002). The grateful disposition: A conceptual and empirical topography. Journal of Personality and Social Psychology, 82, 112-127.

Meier, L. y Oros, L. B. (2012). Percepción de las prácticas parentales y experiencia de emociones positivas en adolescentes. Revista de Psicología, 9(16), 73-84

Mestre Escrivá, V., Pérez Delgado, E., Frías Navarro, D. y Samper, P. (1999). Instrumentos de evaluación de la empatía. En E. Pérez Delgado y V. Mestre (Eds.), Psicología moral y crecimiento personal (pp. 181-190). Edit. Ariel, Barcelona.

Moyano, N. (2011). Gratitud en la Psicoterapia Cognitiva: elementos para su inclusión. Psicodebate. Psicología, Cultura y Sociedad, 11, 9-26.

Mustaca, A. E., Kamenetzky, G. \& Vera-Villarroel, P. (2010). Relaciones entre Variables Positivas y Negativas en una Muestra de Estudiantes Argentinos. Revista Argentina de Clínica Psicológica, 19(3), 227-235.

Namakforoosh M. N. (2000). Metodología de la investigación. México: Limusa.

Oros, L. B. (2006). Evaluación de la simpatía en niños de 6 y 7 años de edad. Libro de Ponencias del X Congreso Nacional de Psicodiagnóstico (345-349). Buenos Aires: ADEIP.

Oros, L. B. (2008a). Promoviendo la serenidad infantil en el contexto escolar. Experiencias preliminares en una zona de riesgo ambiental. Revista Interdisciplinaria, 25(2), 181-196.

Oros, L. B. (2008b). Avances metodológicos en evaluación de emociones positivas en niños en riesgo social. Revista EV ALUAR, 8, 20-33.

Oros, L.B. (2009a). Impacto de una intervención continua para estimular las emociones positivas en niños y niñas afectados por la pobreza. En M. C. Richaud de Minzi y J. E. Moreno (Eds.), Recientes desarrollos iberoamericanos en investigación en Ciencias del Comportamiento (pp.243-255), Buenos Aires: CIIPME-CONICET. 
Oros, L. B. (2009b). El valor adaptativo de las emociones positivas. Una mirada al funcionamiento psicológico de los niños pobres. Revista Interamericana de Psicología, 43(2), 288-296.

Oros, L. B. (2011). Nuevas contribuciones para el desarrollo de emociones positivas en niños. En M. C. Richaud de Minzi \& V. Lemos (Eds.) Psicología y otras Ciencias del Comportamiento. Compendio de Investigaciones Actuales (63-78) Entre Ríos: Universidad Adventista del Plata.

Oros, L.B. \& Richaud de Minzi, M.C. (2011). Cómo incentivar las emociones positivas en los niños. Propuestas para la escuela y la familia. Buenos Aires: ACESUAP.

Porro-Conforti, M. L. \& Andrés, M. L. (2011). Tipo de expresividad emocional y tendencia a la supresión de la expresión emocional en pacientes diagnosticados con cáncer. Psiencia. Revista latinoamericana de ciencia psicológica, 3(1) $10-18$.

Regner (2009a). Emociones positivas como recurso psicológico para el afrontamiento de la amenza (Tesis doctoral no publicada). Pontificia Universidad Católica Argentina. Buenos Aires, Argentina.

Regner, E. (2009b). Compasión y Gratitud, emociones empáticas que elicitan las conductas prosociales. En M. Richaud \& J. E. Moreno, J. E. (Comp.), Investigación en Ciencias del Comportamiento. Avances Iberoamericanos (pp. 281-298). Buenos Aires: CIIPME-CONICET.

Regner, E. (2011, Julio). Positive Emotions, Emotion Regulation and Prosocial Behavior. Poster Session presented at Second World Congress on Positive Psychology. Philadelphia, Pennsylvania, USA.

Ren, J., Hu, L., Zhang, H. \& Huang, Z. (2010). Implicit Positive Emotion Counteracts Ego Depletion. Social Behavior and Personality, 38(7), 919-928.
Reyes-García, V., Gravlee, C. C., McDade, T. W., Huanca, T., Leonard, W. R., \& Tanner, S. (2010). Cultural Consonance and Psychological WellBeing. Estimates Using Longitudinal Data from an Amazonian Society. Culture, Medicine and Psychiatry, 34, 186-203.

Roberts, K. \& Cunningham, G. Serenity: Concept analysis and measurement. Educational Gerontology 16(6), 577-589.

Salgado Levano, A. C. (2009). Felicidad, resiliencia y optimismo en estudiantes de colegios nacionales de la ciudad de Lima. Liberabit, 15(2), 33-141.

Schmidt, C. (2008). Construcción de un cuestionario de emociones positivas en población entrerriana. Revista Iberoamericana de Diagnóstico y Evaluación Psicológica, 26(2), 117 - 139.

Schulz de Begle, A. K. (2009). Correlatos emocionales y relacionales de las habilidades sociales en los niños argentinos. En M. C. Richaud \& J. E. Moreno (Eds.), Investigación en Ciencias del Comportamiento (351-379). Buenos Aires: CIIPME-CONICET.

Schulz de Begle, A. K., Lemos, V. N., \& Richaud de Minzi, M. C. (2009, junio). Validación de la Positive Affect and Negative Affect Schedule Versión Niños (Panas-C) en la población infantil argentina. Comunicación presentada en el XXXII Congreso Interamericano e Psicología, Guatemala

Vera Noriega, J. A., Laborín Álvarez, J. F., Córdova Moreno, M. A. \& Parra Armenta. (2007). Bienestar subjetivo: comparación en dos contextos culturales. Psicología para América Latina. Revista electrónica internacional para América Latina, 11. Recuperado de http://psicolatina.org/11.

(Articulo recibido: 17-9-2012; revisado: 23-11-2012; aceptado: 10-12-2012)

\section{Anexo: CUESTIONARIO INFANTIL de EMOCIONES POSITIVAS}

Por favor responde las frases que aparecen más abajo haciendo una cruz (x) en la casilla que corresponda: Si, Más o menos, o No.

No hay respuestas correctas o incorrectas, sólo es importante que seas honesto. Muchas Gracias.

1. Soy una persona alegre

2. Estoy agradecido con varias personas, por lo que hacen por mí

3. La mayor parte de los días me siento en paz

4. Valoro cuando los demás me ayudan

5. Soy bastante tranquilo

6. Me pongo muy mal si veo que alguien se lastima

7. Si veo llorar a un/a nene/a me dan ganas de llorar a mí también

8. Me quiero mucho a mí mismo

9. Soluciono mis problemas con mucha tranquilidad

10. Me gusta devolver favores

11. Cuando alguien está solo y aburrido me dan ganas de acercarme y jugar con él

12. Me divierto mucho con las cosas que hago

13. Soy muy feliz

14. Casi siempre estoy relajado

15. Siempre que puedo, devuelvo los favores que recibo

16. Siento que soy muy valioso

17. Aunque tenga problemas, igual mantengo la calma

18. Casi siempre la paso bien

19. Me quedo tranquilo aunque no pueda hacer lo que me gusta

20. Siento que soy importante

21. Si alguien está llorando me dan ganas de abrazarlo o consolarlo

22. Me gusta agradecerle a la gente

23. Casi siempre estoy contento/a 